\title{
Pictures of Blockscale Transport: Effective versus Ensemble Dispersion and Its Uncertainty
}

\author{
Felipe P. J. de Barros ${ }^{\mathrm{a}}$, Marco Dentz ${ }^{\mathrm{b}}$ \\ ${ }^{a}$ Sonny Astani Department of Civil and Environmental Engineering, University of \\ Southern California, Los Angeles, California, USA \\ ${ }^{b}$ Institute of Environmental Assessment and Water Research (IDAEA), Spanish National \\ Research Council (CSIC), Barcelona, Spain
}

\begin{abstract}
Solute transport models tend to use coarse numerical grid blocks to alleviate computational costs. Aside from computational issues, the subsurface environment is usually characterized over a coarse measurement network where only large scale fluctuations of the flow field are captured. Neglecting the subscale velocity fluctuations in transport simulators can lead to erroneous predictions with consequences in risk analysis and remediation. For such reasons, upscaled dispersion coefficients in spatially heterogeneous flow fields must (1) account for the subscale variability that is filtered out by homogenization and (2) be modeled as a random function to incorporate the uncertainty associated with non-ergodic solute bodies. In this work, we examine the low order statistical properties of the blockscale dispersion tensor. The blockscale is defined as the scale upon which the spatially variable flow field is homogenized (e.g. the numerical grid block). Using a Lagrangian framework, we discuss different conceptualizations of the blockscale dispersion tensor. We distinguish effective and ensemble blockscale dispersion, which measure the impact of subscale velocity fluctuations on solute dispersion. Ensemble dispersion quantifies subscale velocity fluctuations between realizations, which overestimates the actual velocity variability. Effective dispersion on the other hand quantifies the actual blockscale velocity variability and thus reflects the impact of subscale velocity fluctuations on mixing and spreading. Based on these concepts, we quantify the impact of subscale velocity fluctuations on solute particle spreading and determine the governing equations for the coarse-grained concentration distributions. We develop analytical and semi-analytical expressions for the average and variance of the blockscale
\end{abstract}


dispersion tensor in 3D flow fields as a function of the structural parameters characterizing the subsurface. Our results illustrate the relevance of the blockscale, the initial scale of the solute body and local-scale dispersion in controlling the uncertainty of the plume's dispersive behavior. The analysis performed in this work has implications in numerical modeling (i.e. grid design) and allows to quantify the uncertainty of the blockscale dispersion tensor.

Keywords: Stochastic Hydrogeology, Blockscale Dispersion, Random Flow Fields, Self-Averaging of Dispersion, Numerical Modeling, Uncertainty Quantification, Solute Mixing and Spreading

\section{Introduction}

Natural porous media are spatially variable and characterized by a distribution of heterogeneity scales. This multi-scale variability is an important factor controlling flow and large scale dispersive behavior of a solute plume. A common approach to model the effects of heterogeneity in solute transport is through the use of dispersion coefficients. Defining dispersion coefficients allows to quantify the impact of the subscale velocity fluctuations on solute spreading. In general, dispersion coefficients are not constant and evolve in time [1, 2] and in many cases, the observed large scale transport displays a non-Fickian behavior [3, 4, 5]. The key question is in determining the subscale relevant to solute dispersion.

The difficulty associated with transport modeling in natural porous media is that an accurate reproduction of the spatial structure of the hydraulic properties at all scales is not achievable within the context of applications. This is mainly because of technological and financial budget constraints [6]. As a consequence, the spatial variability of the hydrogeological properties is characterized over a coarse measurement network. Hence only large scale features of the hydraulic conductivity fluctuations are deterministically captured at the field site and this information will be transferred to the numerical grid of the flow and transport simulator [7, 8, 9, 10, 11, 12]. However, when modeling solute transport, it is important to account for subscale variability since velocity fluctuations at scales smaller than the solute body tend to distort the plume and create new concentration gradients. Thus, the subscale is defined by the resolution scale imposed by measurements as well as computational resources (i.e. numerical grid). Neglecting the small scale 
variability not captured directly on the numerical grid can potentially lead to erroneous transport predictions and consequently, wrong decision making when managing contaminated sites.

To compensate for the subscale variability in solute transport modeling, Rubin et al. [11] developed the concept of block-effective dispersion. The main idea proposed in Rubin et al. [11, 13] consists in developing an ensemble based block-effective dispersion tensor that accounts for the homogenized blockscale variability without duplicating the large scale heterogeneity of the conductivity field directly captured on the numerical grid. This concept allows flexibility in numerical grid design without neglecting the dispersive flux of the unmodeled variability and alleviates the computational burden associated with fine grid resolution numerical simulations [14, 15, 16, 17, 18. Similar blockscale dispersion analysis was performed through the use of volume averaging [19, 20].

Theoretical expressions for the ensemble dispersion tensor were obtained by fully homogenizing the heterogeneous porous formation [1, 21, 22, 23, 24]. The ensemble dispersion theory (also known as the macrodispersion theory) in the aforementioned works is valid under ergodic conditions, e.g., when the dimensions of solute body perpendicular to the mean flow direction is much larger the correlation scale of heterogeneity. However, in many applications, the characteristic solute plume dimensions are comparable to the scale of heterogeneity thus leading to non-ergodic transport conditions; this implies that dispersion coefficients are prone to uncertainty. With the aim of studying dispersion under non-ergodic conditions, effective dispersion coefficients were developed to predict the expected dispersion behavior within a single realization of the hydraulic conductivity field [e.g., 25, 26, 27, 28, 29, 30, 31, 32]. The effective dispersion tensor converges to the ensemble dispersion tensor when the solute plume samples the full heterogeneous structure of the geological formation. Furthermore, as discussed in the literature, the effective dispersion coefficient for a point source quantifies mixing whereas the ensemble dispersion spreading [33, 32, 34].

Therefore, in order to accurately describe the dispersion behavior of a solute plume in random flow fields, the dispersion tensor must: (1) be modeled as a random function since the evolution of the non-ergodic solute body is not deterministic; and (2) must incorporate the subscale variability of the velocity field that is lost due to homogenization (such as in a numerical grid). Hence, dispersion tensor quantifying large-scale transport is a random function and should be evaluated at the blockscale. To address this challenge, 
de Barros and Rubin [35] developed a modeling framework that treats the dispersion tensor as a random function. The modeling framework proposed by de Barros and Rubin [35] incorporates the scale of the solute body and the scale of the grid block. Under different limiting cases, de Barros and Rubin [35] showed that the blockscale dispersion random function model recovers the conditions explored in the past. The authors [35] developed expressions for the blockscale dispersion variance for a finite size solute body released in a stratified geological formation under pure advective transport and, through the minimum relative entropy principle, inferred a model for the probability density function of the blockscale dispersion tensor. Within the context of a fully homogenized aquifer system (e.g., infinite blockscale), Dentz and de Barros [36] derived expressions for the dispersion variance tensor in two- and three-dimensional statistically anisotropic random flow fields in the presence of local-scale dispersion. The evolution of the dispersion variance provides information on the mixing efficiency of the transport processes. As the solute plume becomes better mixed, the dispersion variance decreases since the variability between realization reduces. The authors [36] showed that the self-averaging behavior of the solute plume strongly depends on the flow dimensionality and the geometrical properties of the injection source. Selfaveraging implies that the dispersion variance tends to zero for large times (e.g. asymptotic ergodicity). The self-averaging properties of the fully upscaled dispersion coefficient was also studied in a two-dimensional stratified random flow field [37] and multi-Gaussian random flows [38, 39].

The aim of this work is to develop and discuss a dispersion concept that reflects the actual blockscale velocity variability and its impact on solute mixing and spreading. In addition, we quantify the sample-to-sample fluctuations of the blockscale dispersion through its variance. We provide a rational framework that allows (1) to determine dispersion coefficients in coarse-grained models and (2) quantify the corresponding uncertainty in the blockscale dispersion tensor. The mean and variance of the dispersion coefficient are determined as a function of the blockscale, the characteristic size of the solute plume, statistical anisotropy ratio of the heterogeneous permeability field and local dispersion. We develop novel semi-analytical expressions for the first two statistical moments of the blockscale dispersion in 3D flow fields under finite Péclet conditions. Our results shed new light on how the characteristic length scales defining the transport problem control the level of uncertainty in transport predictions. Specifically, we illustrate the potential of the scale of the homogenizing region in reducing the uncertainty of 
dispersion coefficients.

\section{Basics and Methodology}

\subsection{Flow and Transport}

The physical problem investigated in this work consists of a $d$-dimensional steady state, spatially heterogeneous, incompressible Darcy flow field. The fluctuation of the flow field stems from the heterogeneity of the geological medium's hydraulic conductivity. The hydraulic conductivity $K(\mathbf{x})$ is locally isotropic and spatially variable in $\mathbf{x}=\left(x_{1}, \ldots, x_{d}\right)^{T}$. Flow is assumed to be uniform-in-the-mean along the $x_{1}$ direction. For the purpose of this work, we consider flow to be divergence free in the absence of sinks/sources and far from boundary effects. Furthermore, the effective porosity $\phi$ of the medium is assumed to be constant. The divergence-free velocity field $\mathbf{v}(\mathbf{x})$ is determined via Darcy's law and the flow equation

$$
\mathbf{v}(\mathbf{x})=-K(\mathbf{x}) \nabla h(\mathbf{x}) / \phi, \quad \nabla \cdot[K(\mathbf{x}) \nabla h(\mathbf{x})]=0 .
$$

where $h(\mathbf{x})$ is the hydraulic head.

An inert dissolved solute is instantaneously released at time $t=t_{0}$ and the plume is advected and dispersed according to the governing equation

$$
\frac{\partial c(\mathbf{x}, t)}{\partial t}+\mathbf{v}(\mathbf{x}) \cdot \nabla c(\mathbf{x}, t)=\nabla \cdot[\mathbf{D} \nabla c(\mathbf{x}, t)]
$$

where $c(\mathbf{x}, t)$ is the resident concentration of the dissolved passive solute. The initial solute distribution is $c\left(\mathbf{x}, t=t_{0}\right)=\rho(\mathbf{x})$ and the local-scale dispersion tensor $\mathbf{D}$ is assumed to be diagonal, e.g. $D_{i j}=D_{i i} \delta_{i j}$ with $\delta_{i j}$ denoting Kronecker's delta. For simplicity, we assume $t_{0}=0$

Solute transport can be described equivalently in terms of the equations of motion of solute particles by the Langevin equation [40]

$$
\frac{d \mathbf{x}(t \mid \mathbf{a})}{d t}=\mathbf{v}[\mathbf{x}(t \mid \mathbf{a})]+\sqrt{2 \mathbf{D}} \cdot \boldsymbol{\xi}(t)
$$

where $\boldsymbol{\xi}(t)$ denotes a Gaussian white noise with zero mean and covariance $\left\langle\xi_{i}(t) \xi_{j}\left(t^{\prime}\right)\right\rangle=\delta_{i j} \delta\left(t-t^{\prime}\right)$ with $\delta_{i j}$ the Kronecker delta and $\delta(t)$ corresponding to the Dirac delta. In this work, $\boldsymbol{\xi}(t)$ will be termed local noise since it is related to the local scale dispersion tensor $\mathbf{D}$ [5]. The angular brackets $\langle\cdot\rangle$ denote the average over all noise realizations and the particle position 
is represented by $\mathbf{x}(t \mid \mathbf{a})$ with the initial position $\mathbf{x}\left(t=t_{0} \mid \mathbf{a}\right)=\mathbf{a}$. Within the context of particle motion, $\rho(\mathbf{a})$ corresponds to the distribution of initial particle positions. The solute distribution $c(\mathbf{x}, t)$ is expressed in terms of the particle trajectories $\mathbf{x}(t \mid \mathbf{a})$ as

$$
c(\mathbf{x}, t)=\int\langle\delta[\mathbf{x}-\mathbf{x}(t \mid \mathbf{a})]\rangle \rho(\mathbf{a}) d \mathbf{a} .
$$

In the following, we denote the Green function $\langle\delta[\mathbf{x}-\mathbf{x}(t \mid \mathbf{a})]\rangle$ by

$$
g(\mathbf{x}, t \mid \mathbf{a})=\langle\delta[\mathbf{x}-\mathbf{x}(t \mid \mathbf{a})]\rangle .
$$

The flow and transport equations (11)-(3) fully describe passive solute transport in the heterogeneous porous medium characterized by the spatially varying hydraulic conductivity $K(\mathbf{x})$. Once $\mathbf{v}(\mathbf{x})$ is obtained from (11), solute transport can be quantified by solving the advection-dispersion equation (2) or the Langevin equation (3). The numerical solution of (2) on a discretized flow domain consisting of grid blocks of characteristic size $\lambda$ implies the loss of velocity variability on scales below $\lambda$. The same applies to the concentration distribution and particle trajectories that are obtained from a smoothed, e.g. interpolated, flow field. Only in the case of a discretization scale of the order of the smallest heterogeneity scale, i.e. a highly refined numerical mesh, does the numerical solution reproduce the full heterogeneity induced flow and transport behavior. However, for a fine discretization of the flow domain, the computational burden is heavy, specially in the context of stochastic simulations where the Monte Carlo framework is commonly adopted [e.g., 41]. Thus, limited computational resources impose a constraint on the design of the numerical mesh and coarse grid blocks are generally used. On the other hand, it is generally not possible to have detailed knowledge on the fluctuation behavior of $K(\mathbf{x})$ over various orders of magnitude. As a consequence, the resolution of $K(\mathbf{x})$ relies on a given practical characterization scale (for example, a measurement grid) and the spatial resolution of the measurement device [8].

Thus, it is necessary to account for the impact of velocity fluctuations below the grid or characterization scale $\lambda$ on large scale transport. Solute transport in the coarse scale $\lambda$ renders concentration distributions and particle trajectories that are smoother than the ones obtained from the fully resolved fine scale velocity field. This leads to an inaccurate description of dispersion and large scale transport [42, 11, 43, 35], with implications in human health risk estimation and decision making [44, 45]. The challenge lies in 
compensating the random subscale velocity variability, that is homogenized at a scale smaller than $\lambda$, through its impact of effective solute dispersion. We approach this problem using stochastic modeling in conjunction with a spatial filtering methodology in order to quantify subscale velocity fluctuations in a systematic manner.

\subsection{Stochastic Model}

The spatially varying hydraulic conductivity field is considered a realization of a stationary and ergodic random field. Specifically, the log-hydraulic conductivity, $Y(\mathbf{x})=\ln [K(\mathbf{x})]$, is modeled as a multi-Gaussian random space function [6]. The randomness in the medium properties is mapped onto the flow velocity $\mathbf{v}(\mathbf{x})$ through the Darcy equation (1), which then is passed on to solute and particle transport via (2) and (3).

The multi-Gaussian $Y(\mathbf{x})$ is characterized by its constant mean $\overline{Y(\mathbf{x})}=$ $\bar{Y}$, variance $\sigma_{Y}^{2}$, correlation length scale $l_{i}$ (with $i=1, \ldots, d$ ) and two-point spatial covariance $C_{Y}(\mathbf{r})=\overline{Y^{\prime}(\mathbf{x}) Y^{\prime}(\mathbf{x}+\mathbf{r})}$ where $\mathbf{r}$ is the lag distance between two distinct locations within the flow domain and $Y^{\prime}(\mathbf{x})=\bar{Y}-Y^{\prime}(\mathbf{x})$ is the log-conductivity fluctuation. The overbar denotes the ensemble average over all realizations of $Y(\mathbf{x})$. In this work, the Fourier transform of $Y^{\prime}(\mathbf{x})$ is denoted by $\tilde{Y}^{\prime}(\mathbf{k})$ where $\mathbf{k}$ represents the wave number. The Fourier transform of a function $\varphi(\mathbf{x})$ is defined by

$$
\begin{aligned}
& \widetilde{\varphi}(\mathbf{k})=\int_{-\infty}^{\infty} \exp (\imath \mathbf{k} \cdot \mathbf{x}) \varphi(\mathbf{x}) d \mathbf{x} \\
& \varphi(\mathbf{x})=\int_{-\infty}^{\infty} \exp (-\imath \mathbf{k} \cdot \mathbf{x}) \widetilde{\varphi}(\mathbf{k}) \frac{d \mathbf{k}}{(2 \pi)^{d}} .
\end{aligned}
$$

where $\imath$ is the imaginary unit. Here and in the following, Fourier transformed quantities are marked by a tilde. Furthermore, we employ the short-hand notation

$$
\int_{k}[\cdot] \equiv \int_{-\infty}^{\infty}[\cdot] \frac{d \mathbf{k}}{(2 \pi)^{d}}
$$

By making use of Darcy's law and representing the conductivity by a Taylor series expansion about its mean value $\bar{Y}$, we can obtain an approximation for the steady state velocity field [46, 6]. First-order perturbation theory in 
the log-conductivity fluctuations gives for the velocity field $\mathbf{v}(\mathbf{x})$

$$
v_{i}(\mathbf{x})=\bar{v} \delta_{i 1}+\bar{v} \int_{-\infty}^{\infty} \exp (-i \mathbf{k} \cdot \mathbf{x}) p_{i}(\mathbf{k}) \tilde{Y}(\mathbf{k}) \frac{d \mathbf{k}}{(2 \pi)^{d}}
$$

where $\mathbf{k}$ is the wave number. We assume that the mean hydraulic gradient is aligned with the 1 -direction of the coordinate system, $\overline{\nabla h}=G \mathbf{e}_{1}$. The mean velocity is $\bar{v}=G K_{g}$, where $K_{g}=\exp (\bar{Y})$ corresponds to the geometric mean hydraulic conductivity. The projectors $p_{i}(\mathbf{k})$ are defined by $p_{i}(\mathbf{k})=$ $\delta_{i 1}-k_{1} k_{i} / \mathbf{k}^{2}$ and guarantee that $\nabla \cdot \mathbf{v}(\mathbf{x})=0$ through $\sum_{i=1}^{d} k_{i} p_{i}(\mathbf{k})=0$.

The velocity field (9) is a linear functional of the multi-Gaussian $Y^{\prime}(\mathbf{x})$ and therefore, corresponds to a multi-Gaussian random space function itself. Its mean is given by $\overline{\mathbf{v}(\mathbf{x})}=\bar{v} \mathbf{e}_{1}$ and the velocity fluctuations $v_{i}^{\prime}(\mathbf{x})=v_{i}(\mathbf{x})-\bar{v} \delta_{i 1}$ are expressed in the second term on the right hand side of (9). The covariance of their Fourier transforms is

$$
\overline{\widetilde{v}_{i}^{\prime}(\mathbf{k}) \widetilde{v}^{\prime}\left(\mathbf{k}^{\prime}\right)}=(2 \pi)^{2} \delta\left(\mathbf{k}+\mathbf{k}^{\prime}\right) \widetilde{C}_{i j}(\mathbf{k}), \quad \text { with } \quad \widetilde{C}_{i j}(\mathbf{k})=p_{i}(\mathbf{k}) p_{j}(\mathbf{k}) \widetilde{C}_{Y}(\mathbf{k})
$$

where $\widetilde{C}_{i j}(\mathbf{k})$ is the velocity covariance function in Fourier space. The Dirac delta in equation (10) is a consequence of the stationarity of the random field $Y(\mathbf{x})$.

\subsection{Solute Transport at the Blockscale}

Solute transport is investigated from the Lagrangian point of view starting from the Langevin equation (3). We begin by defining a coarse grained velocity field and the corresponding subscale velocity fluctuations, which in this framework are incorporated into a suitably defined mesoscopic noise term.

The spatial velocity fluctuations are separated in the contributions below and above the coarse graining scale $\lambda$ in order to determine the dispersion effect due to subscale velocity fluctuations. The coarse-grained velocity field $\mathbf{v}_{>}(\mathbf{x})$ is defined by spatial filtering as

$$
\mathbf{v}_{>}(\mathbf{x})=\int d \mathbf{r} \mathcal{F}(\mathbf{x}-\mathbf{r}) \mathbf{v}(\mathbf{r})
$$

with $\mathcal{F}(\mathbf{x})$ corresponding to a suitably chosen spatial filter 47,48, 49, 9, 11 , 12, 50]. This coarse-graining operation reads in Fourier space as

$$
\widetilde{\mathbf{v}}_{>}(\mathbf{k})=\widetilde{\mathcal{F}}(\mathbf{k}) \widetilde{\mathbf{v}}(\mathbf{k}),
$$



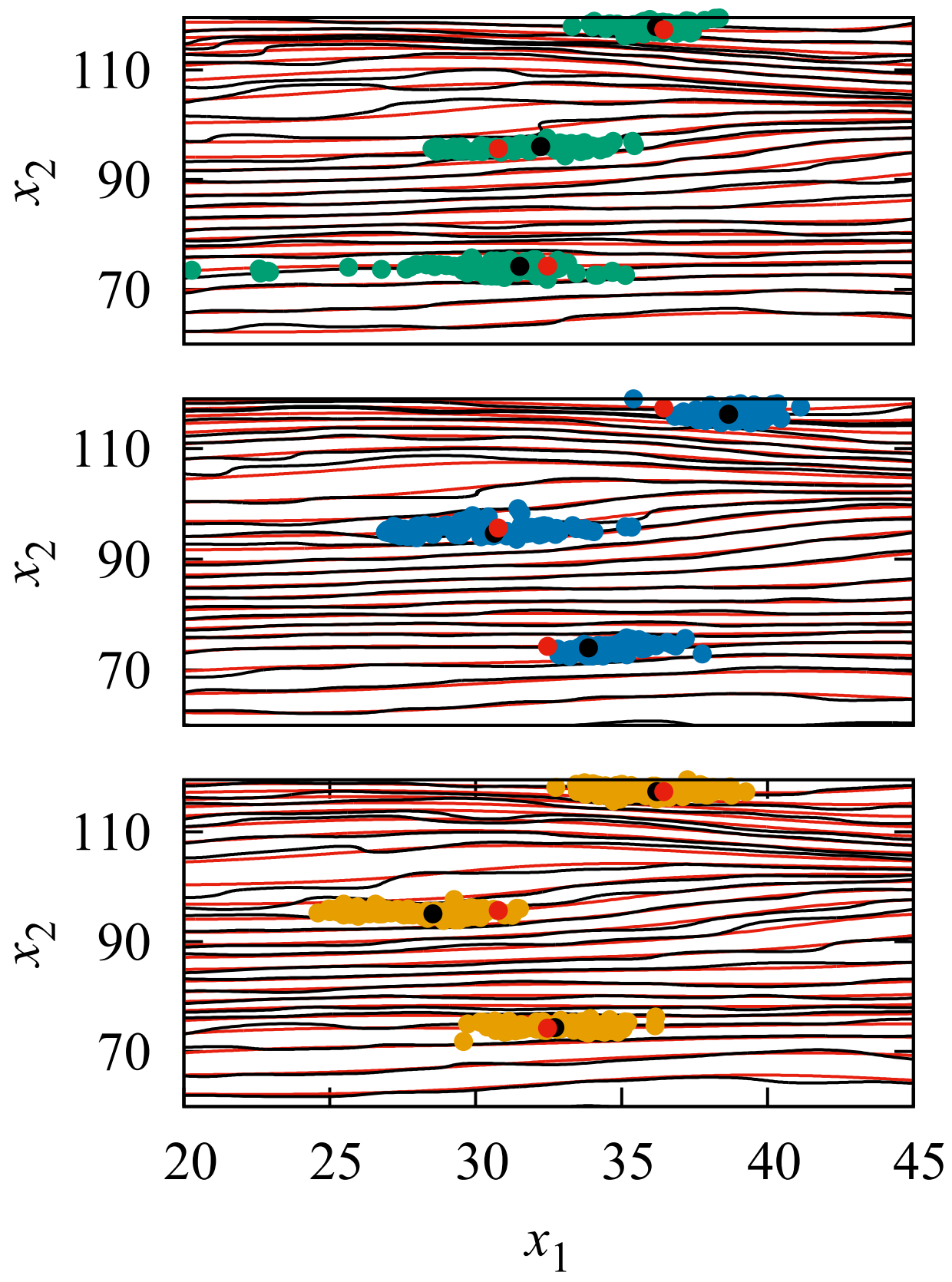

Figure 1: Illustration of particles plumes in different realizations of $\mathbf{v}(\mathbf{x})$ characterized by the same coarse-grained $\mathbf{v}_{>}(\mathbf{x})$. The solid black lines illustrate the streamlines of the fully variable velocity field $\mathbf{v}(\mathbf{x})$ and the solid red lines the streamlines of the coarse-grained velocity field $\mathbf{v}_{>}(\mathbf{x})$. The black dots denote the center of mass of the respective local particle distribution in the individual realization, while the red dots show the center of mass position obtained from the coarse-grained velocity $\mathbf{v}_{>}(\mathbf{x})$. 
where the spatial filter $\mathcal{F}(\mathbf{x})$ is normalized, i.e.,

$$
\int \mathcal{F}(\mathbf{x}) d \mathbf{x}=1, \quad \widetilde{\mathcal{F}}(\mathbf{k}=\mathbf{0})=1 .
$$

The subscale scale velocity fluctuations are defined correspondingly by

$$
\mathbf{v}_{<}(\mathbf{x})=\mathbf{v}(\mathbf{x})-\mathbf{v}_{>}(\mathbf{x})
$$

Thus, the ensemble mean of the coarse grained flow velocity (11) is equal to the mean flow velocity while the subscale fluctuations are, in average, zero,

$$
\overline{\mathbf{v}_{>}(\mathbf{x})}=\overline{\mathbf{v}}, \quad \overline{\mathbf{v}_{<}(\mathbf{x})}=\mathbf{0}
$$

Figure 1 illustrates three random velocity fields $\mathbf{v}(\mathbf{x})$ characterized by the same coarse-grained velocity field $\mathbf{v}_{>}(\mathbf{x})$.

Next, we rewrite the fine scale Langevin equation (3) in the following form

$$
\frac{d \mathbf{x}(t \mid \mathbf{a})}{d t}=\mathbf{v}_{>}[\mathbf{x}(t \mid \mathbf{a})]+\boldsymbol{\zeta}(t \mid \mathbf{a})
$$

where the mesoscale noise $\boldsymbol{\zeta}(t \mid \mathbf{a})$ is defined in terms of the subscale velocity fluctuations and the local noise term as

$$
\boldsymbol{\zeta}(t \mid \mathbf{a})=\mathbf{v}_{<}[\mathbf{x}(t \mid \mathbf{a})]+\sqrt{2 \mathbf{D}} \cdot \boldsymbol{\xi}(t) .
$$

The mesoscale noise $\boldsymbol{\zeta}(t \mid \mathbf{a})$ represents the impact of subscale heterogeneity on coarse-scale solute dispersion. Notice that $\boldsymbol{\zeta}(t \mid \mathbf{a})$ is a multi-Gaussian noise due to the Gaussianity of $\boldsymbol{\xi}(t)$ and $\mathbf{v}(\mathbf{x})$ (i.e. stochastic process for the velocity fluctuations at the subscale). Figure 1 illustrates particle distributions in different realizations of $\mathbf{v}(\mathbf{x})$ for the same coarse-grained $\mathbf{v}_{>}(\mathbf{x})$. The effective noise quantifies fluctuations about the coarse-grained velocity $\mathbf{v}_{>}(\mathbf{x})$. Note that the center of mass of the particle distribution in individual realization does in general not coincide with the coarse-grained center of mass (compare the locations of the black dots and the red dots in Figure 1). In fact, as discussed in the following, the statistical properties of $\boldsymbol{\zeta}(t \mid \mathbf{a})$ depend on the order in which the noise and ensemble averages are performed. In the upcoming section, we will show how the order of the averaging operation leads to distinct conceptualizations of the blockscale dispersion tensor. 


\section{Blockscale Dispersion Concepts}

In the previous section, we defined the mesoscale noise (17), in order to quantify the dispersion effect due subscale velocity fluctuations. It comprises local noise, which models the impact of local dispersion, as well as the filtered velocity fluctuations. Thus, $\boldsymbol{\zeta}(t \mid \mathbf{a})$ depends on the local noise, on the realization of the flow velocity as well as on the initial position a of the respective solute particle. The statistical properties of $\boldsymbol{\zeta}(t \mid \mathbf{a})$, specifically, its covariance, depend on the order in which the averages over the noise and flow ensemble are taken, and which the integral over the initial particle positions is performed. In the following, we discuss two different averaging procedure, which define the statistical properties of the mesoscale noise. These are as follows:

1. The first procedure is termed ensemble noise average. It defines the covariance of $\boldsymbol{\zeta}(t \mid \mathbf{a})$ with respect to its noise and flow ensemble average. It quantifies velocity fluctuations with respect to the coarse-grained velocity $\mathbf{v}(\mathbf{x})$ between realizations of the finescale velocity fluctuations $\mathbf{v}_{<}(\mathbf{x})$. As illustrated in Figure 2, the center of mass position of a particle distribution in a realization of $\mathbf{v}(\mathbf{x})$ does not necessarily coincide with $\mathbf{v}_{>}(\mathbf{x})$. Thus, the ensemble blockscale dispersion quantifies an artificial ensemble effect due to center of mass fluctuation between realizations of $\mathbf{v}_{<}(\mathbf{x})$.

2. The second procedure is termed effective noise average. It measures the dispersion effect of velocity fluctuations with respect to the actual mean velocity in a single realization of $\mathbf{v}_{<}(\mathbf{x})$, which does not, in general, coincide with the coarse-grained $\mathbf{v}_{>}(\mathbf{x})$, see also Figure 2 . Thus the effective noise average represents genuine subscale velocity fluctuations.

Based on these concepts, we define two surrogate processes that are formalized by Langevin equations, which are characterized by the respective ensemble and effective noises. These noises are correlated Gaussian processes characterized by the respective covariances. From these Langevin equations, we then derive the corresponding advection-dispersion equations that govern the evolution of the coarse-scale solute distributions in the two different dispersion models.

\subsection{Blockscale Dispersion Based on the Ensemble Noise Average}

The first averaging procedure employed and investigated is the ensemble noise average of $\boldsymbol{\zeta}(t \mid \mathbf{a})$. The ensemble noise average of the mesoscale noise 

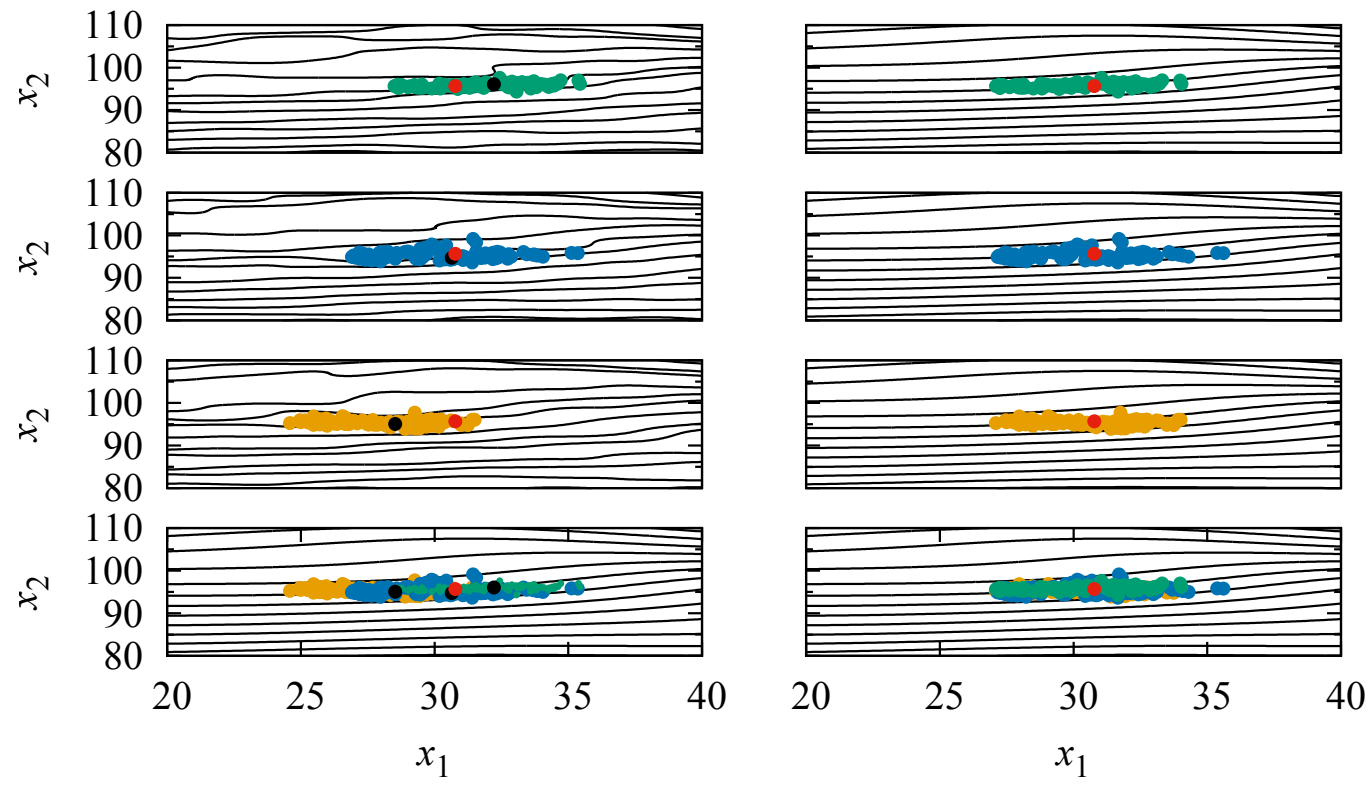

Figure 2: Illustration of the concepts of ensemble and effective blockscale dispersion. Solid black lines illustrate streamlines. (Left panel) Evolution of solute plumes (rows 1-3 from top to bottom) in three different realizations of $\mathbf{v}(\mathbf{x})$ characterized by the same $\mathbf{v}_{>}(\mathbf{x})$. The red dot denote the center of mass position obtained from the coarse-grained velocity field and the black dots denote the actual center of mass positions in the particular realization of $\mathbf{v}(\mathbf{x})$. The bottom figure illustrates the ensemble blockscale dispersion concept, which quantifies fluctuations of the center of mass velocity in individual realizations with respect to the coarse-grained center of mass velocity. (Right panel) Solute plumes centered with respect to the coarse-grained center of mass position in the coarse-grained velocity field. The bottom figure illustrates the effective blockscale dispersion concept, which quantifies the velocity fluctuations with respect to the center of mass velocity in individual realizations. 
considers the covariance of the mesoscale noise with respect to the ensemble noise average

$$
\overline{\langle\boldsymbol{\zeta}(t \mid \mathbf{a})\rangle}=\mathbf{0} .
$$

Thus, we define the ensemble covariance of the mesoscale noise by

$$
C_{i j}^{e n s}\left(t, t^{\prime}\right)=\overline{\left\langle\zeta_{i}(t \mid \mathbf{a}) \zeta_{j}\left(t^{\prime} \mid \mathbf{a}\right)\right\rangle} .
$$

The ensemble covariance quantifies the velocity covariance with respect to the coarse-grained velocity $\mathbf{v}_{>}(\mathbf{x})$. The expression on the right hand side of (19) can be expanded such that we obtain for the ensemble covariance

$$
\begin{aligned}
& C_{i j}^{e n s}\left(t-t^{\prime}\right)=2 D_{i i} \delta_{i j} \delta\left(t-t^{\prime}\right)+\int_{k} \int_{k^{\prime}} \int_{k^{\prime \prime}} \mathcal{A}(\mathbf{k}) \mathcal{A}\left(\mathbf{k}^{\prime}\right) \\
& \times{\widetilde{v_{i}}(\mathbf{k}) \widetilde{v}_{j}\left(\mathbf{k}^{\prime}\right) \widetilde{g}\left(-\mathbf{k}, t-t^{\prime} \mid \mathbf{a}\right) \exp \left(i \mathbf{k}^{\prime \prime} \cdot \mathbf{a}\right) \widetilde{g}\left(-\mathbf{k}^{\prime}-\mathbf{k}^{\prime \prime}, t^{\prime} \mid \mathbf{a}\right)},
\end{aligned}
$$

where Lagrangian stationarity is assumed, $\mathcal{A}(\mathbf{k})=1-\widetilde{\mathcal{F}}(\mathbf{k})$ and the function $\widetilde{g}(\mathbf{k}, t \mid \mathbf{a})$ is the Fourier transform of the Green function $g(\mathbf{x}, t \mid \mathbf{a})=\langle\delta[\mathbf{x}-$ $\mathbf{x}(t \mid \mathbf{a})]\rangle$. As we will see in the following, the ensemble average covariance does not depend on the initial particle position, as desired for an effective framework. The blockscale ensemble dispersion tensor can be evaluated by integrating the ensemble covariance of the mesoscopic noise $C_{i j}^{e n s}$ :

$$
D_{i j}^{e n s}\left(t-t_{0}\right)=\int_{0}^{t-t_{0}} d t^{\prime} C_{i j}^{e n s}\left(t^{\prime}\right),
$$

where $t_{0}$ is the initial time. The dispersion tensor $D_{i j}^{e n s}\left(t-t_{0}\right)$ in (21) is also reported in eq. 4 of Fiori et al. [51]. Given the aforementioned development, we may now define the coarse-grained stochastic process

$$
\frac{d \mathbf{x}(t \mid \mathbf{a})}{d t}=\mathbf{v}_{>}[\mathbf{x}(t \mid \mathbf{a})]+\boldsymbol{\zeta}^{e n s}(t)
$$

where the ensemble noise $\boldsymbol{\zeta}^{\text {ens }}(t)$ is characterized by zero mean $\left\langle\boldsymbol{\zeta}^{\text {ens }}(t)\right\rangle_{\text {ens }}=$ $\mathbf{0}$ and the covariance function $\left\langle\zeta_{i}^{e n s}(t) \zeta_{j}^{e n s}\left(t^{\prime}\right)\right\rangle_{\text {ens }}=\mathcal{C}_{i j}^{e n s}\left(t-t^{\prime}\right)$. The angular brackets with subscript ens denote the ensemble noise average. The average concentration can be determined in terms of the ensemble noise average as

$$
c^{e n s}(\mathbf{x}, t)=\int d \mathbf{a} \rho(\mathbf{a})\langle\delta[\mathbf{x}-\mathbf{x}(t \mid \mathbf{a})]\rangle_{e n s} .
$$


Based on the fact that the mesoscale noise is Gaussian distributed, one can derive the following advection-dispersion equation for $c_{e n s}(\mathbf{x}, t)$

$$
\frac{\partial c^{e n s}(\mathbf{x}, t)}{\partial t}+\mathbf{v}_{>}(\mathbf{x}) \cdot \nabla c^{e n s}(\mathbf{x}, t)-\nabla \cdot\left[\mathbf{D}^{e n s}\left(t-t_{0}\right) \nabla c^{e n s}(\mathbf{x}, t)\right]=0
$$

Note that the dependence of $\mathbf{D}^{e n s}\left(t-t_{0}\right)$ on the initial time $t_{0}$ expresses the non-Markovian nature of the transport processes due to the noise correlation [52, 51]. For the Langevin equation (31), which represents a Markov process, the noise is delta-correlated, and thus the related advection-dispersion equation (2) is independent of the initial time.

\subsection{Blockscale Dispersion Based on the Effective Noise Average}

The effective noise average considers the covariance of the mesoscale noise with respect to the velocity fluctuation averaged over the local noise

$$
\left\langle\zeta_{i}(t \mid \mathbf{a})\right\rangle=\int_{k} \mathcal{A}(\mathbf{k}) \widetilde{v}_{i}(\mathbf{k}) \widetilde{c}(\mathbf{k}, t \mid \mathbf{a})
$$

The effective single realization noise covariance is defined by

$$
\mathcal{C}_{i j}^{e f f}\left(t, t^{\prime}\right)=\left\langle\zeta_{i}(t \mid \mathbf{a}) \zeta_{j}(t \mid \mathbf{a})\right\rangle-\left\langle\zeta_{i}(t \mid \mathbf{a})\right\rangle\left\langle\zeta_{j}\left(t^{\prime} \mid \mathbf{a}\right)\right\rangle .
$$

This means it quantifies the velocity fluctuations with respect to the mean velocity in single realization of the finescale velocity $\mathbf{v}_{<}(\mathbf{x})$, which is given by $\mathbf{v}_{>}(\mathbf{x})+\left\langle\mathbf{v}_{<}(\mathbf{x})\right\rangle$. Notice that the effective noise covariance as an ensemble average does not depend on the initial particle positions. Accordingly, we define now the effective single realization dispersion coefficient as

$$
\mathcal{D}_{i j}^{e f f}(t)=\frac{1}{2} \int_{0}^{t} d t^{\prime}\left[\mathcal{C}_{i j}^{e f f}\left(t, t^{\prime}\right)+\mathcal{C}_{j i}^{e f f}\left(t, t^{\prime}\right)\right] .
$$

It measures the average relative solute and particle dispersion in a single realization of $\mathbf{v}_{<}(\mathbf{x})$. In this sense it may be considered a measure for solute mixing rather than the ensemble dispersion coefficients, which quantify the uncertainty of particle positions within the extended initial plume and between heterogeneity realizations. Similar dispersion conceptualization can be 
found in Fiori [53] and section 3.2 of Dentz and de Barros [36]. The ensemble average $C_{i j}^{e f f}\left(t-t^{\prime}\right)=\overline{\mathcal{C}_{i j}^{e f f}\left(t, t^{\prime}\right)}$ can be written as

$$
\begin{aligned}
C_{i j}^{e f f}\left(t, t^{\prime}\right) & =C_{i j}^{e n s}\left(t, t^{\prime}\right)-\int d \mathbf{a} \rho(\mathbf{a}) \\
& \times \int_{k} \int_{k^{\prime}} \mathcal{A}(\mathbf{k}) \mathcal{A}\left(\mathbf{k}^{\prime}\right) \overline{\widetilde{v}_{i}(\mathbf{k}) \widetilde{v}_{j}\left(\mathbf{k}^{\prime}\right) \widetilde{g}(\mathbf{k}, t \mid \mathbf{a}) \widetilde{g}\left(\mathbf{k}^{\prime}, t^{\prime} \mid \mathbf{a}\right)} .
\end{aligned}
$$

The blockscale effective dispersion coefficient now is given by

$$
D_{i j}^{e f f}(t)=\int_{0}^{t} d t^{\prime} C_{i j}^{e f f}\left(t^{\prime}\right)=\overline{\mathcal{D}_{i j}^{e f f}(t)} .
$$

In order to illustrate the difference between the ensemble and effective dispersion coefficient, we consider the difference between the noise average trajectories and its coarse-grained counter part, $\delta\langle\mathbf{x}(t \mid \mathbf{a})\rangle=\langle\mathbf{x}(t \mid \mathbf{a})\rangle-\left\langle\mathbf{x}_{>}(t \mid \mathbf{a})\right\rangle$, which is equal to

$$
\delta\langle\mathbf{x}(t \mid \mathbf{a})\rangle=\int_{0}^{t} d t^{\prime}\left\langle\boldsymbol{\zeta}\left(t^{\prime} \mid \mathbf{a}\right)\right\rangle
$$

It measures the deviation of the averaged coarse-grained particle trajectory starting at $\mathbf{a}$ with the noise average of $\mathbf{x}(t \mid \mathbf{a})$. The difference between ensemble and effective dispersion coefficients measures the evolution of its variance

$$
\frac{\left[d \overline{\left.\delta\left\langle x_{i}(t \mid \mathbf{a})\right\rangle^{2}\right]_{\rho}}\right.}{d t}=D_{i i}^{e n s}(t)-D_{i i}^{e f f}(t) .
$$

The difference between the ensemble and effective dispersion coefficients measures the evolution of the trajectory uncertainty both due to fluctuations within the initial plume and between the disorder realizations.

As above, we now define an effective coarse grained Langevin equation,

$$
\frac{d \mathbf{x}(t \mid \mathbf{a})}{d t}=\mathbf{v}_{>}[\mathbf{x}(t \mid \mathbf{a})]+\boldsymbol{\zeta}^{e f f}(t)
$$

where the effective noise is characterized zero mean, $\left\langle\boldsymbol{\zeta}^{e f f}(t)\right\rangle_{\text {eff }}=\mathbf{0}$ and the covariance $\left\langle\zeta_{i}^{e f f}(t) \zeta_{j}^{e f f}\left(t^{\prime}\right)\right\rangle_{e f f}=C_{i j}^{e f f}\left(t-t^{\prime}\right)$. The angular brackets with 
subscript eff denote the effective noise average. We define now an effective concentration in terms of the ensemble noise average as

$$
c^{e f f}(\mathbf{x}, t)=\int d \mathbf{a} \rho(\mathbf{a})\langle\delta[\mathbf{x}-\mathbf{x}(t \mid \mathbf{a})]\rangle_{e f f},
$$

where the angular brackets with subscript ens denotes the ensemble noise average. Based on the fact that the mesoscale noise is Gaussian distributed one can derive the following advection-dispersion equation for $c_{e f f}(\mathbf{x}, t)$

$$
\frac{\partial c^{e f f}(\mathbf{x}, t)}{\partial t}+\mathbf{v}_{>}(\mathbf{x}) \cdot \nabla c^{e f f}(\mathbf{x}, t)-\nabla \cdot\left[\mathbf{D}^{e f f}\left(t-t_{0}\right) \nabla c^{e f f}(\mathbf{x}, t)\right]=0 .
$$

As before, the dependence of $\mathbf{D}^{e f f}\left(t-t_{0}\right)$ on the initial time is a manifestation of the non-Markovianity of (32) due to the correlated noise.

\subsection{Self-Averaging of Dispersion}

The stochastic approach renders dispersion coefficients as averages over an ensemble of realizations. Clearly, the dispersion behavior in principle depends on the specific disorder characteristics. This means, that average dispersion coefficients are in general not representative of dispersion in single flow realizations. The fluctuation of the solute plume spreading about its mean value is a measure of heterogeneity-induced uncertainty [26, 54, 55]. However, as more and more of the flow heterogeneity is sampled by the solute plume, e.g., as the transport histories experienced by the scalar in different realizations, converge, the ensemble average is expected to become representative of dispersion in a single realization [35, 36]. If the variance of the dispersion coefficient tends to zero with time, then the dispersion observable is self-averaging [56]. More details on self-averaging behavior of solute spreading in spatially heterogeneous porous media can be found in the literature [26, 35, 36, 37, 38, 39, 57, 58].

The self-averaging behavior [56] is measured by the variance of the single realization dispersion coefficients and their ensemble averaged counterparts. Thus, we have for the effective dispersion coefficients the variances

$$
\overline{\delta \mathcal{D}_{i j}^{e f f}(t)^{2}}=\overline{\left[\mathcal{D}_{i j}^{e f f}(t)-D_{i j}^{e f f}(t)\right]^{2}} .
$$

Expressions for the fully upscaled (e.g. $\lambda \rightarrow \infty$ ) dispersion variance can be found in Dentz and de Barros [36] and asymptotic dispersion variance 
results based on work of Fiori [29] are also reported in Appendix C of Wood et al. [20]. In the following section, we develop explicit perturbation theory expressions for the ensemble and effective blockscale dispersion coefficients as well as the dispersion variances.

\section{Expressions for the Blockscale Dispersion Mean and Variance}

\subsection{Perturbation Theory}

The perturbation theory expressions for the ensemble and effective dispersion coefficients are obtained straightforwardly from (21) and (29) by substituting $\widetilde{g}(\mathbf{k}, t \mid \mathbf{a})$ by

$$
\widetilde{g}_{0}(\mathbf{k}, t \mid \mathbf{a})=\exp \left(-\mathbf{k} \cdot \mathbf{D k}+\imath \bar{v} k_{1} t\right) \exp (\imath \mathbf{k} \cdot \mathbf{a}) .
$$

with $\imath$ corresponding to the imaginary unit. After some algebraic manipulations, we obtain the expressions for the mean dispersion coefficients:

$$
\begin{aligned}
& D_{i j}^{e n s}(t)=D_{i i} \delta_{i j}+\int_{0}^{t} d t^{\prime} \int_{k} \mathcal{A}(\mathbf{k}) \mathcal{A}(-\mathbf{k}) \widetilde{C}_{i j}(\mathbf{k}) \widetilde{g}_{0}\left(-\mathbf{k}, t-t^{\prime} \mid \mathbf{0}\right), \\
& D_{i j}^{e f f}(t)=D_{i j}^{e n s}(t)-\int_{0}^{t} d t^{\prime} \int_{k} \mathcal{A}(\mathbf{k}) \mathcal{A}(-\mathbf{k}) \widetilde{C}_{i j}(\mathbf{k}) \widetilde{g}_{0}(\mathbf{k}, t \mid \mathbf{0}) \widetilde{g}_{0}\left(-\mathbf{k}, t^{\prime} \mid \mathbf{0}\right) .
\end{aligned}
$$

with $\widetilde{C}_{i j}$ given in equation (10). In order to determine perturbation theory expressions for the dispersion variances, see (35), we need to expand the single realization quantities up to first order in the velocity fluctuations. To this end, we consider (27) as well as the expression (26) for the corresponding covariance functions. Up to first order in the velocity fluctuations, we obtain explicitly,

$$
\begin{aligned}
\mathcal{D}_{i j}^{e f f}(t) & =D_{i i} \delta_{i j} \\
& -\int_{k} \imath g_{0}(\mathbf{k}, t \mid \mathbf{0}) \widetilde{\rho}(\mathbf{k}) \mathcal{A}(\mathbf{k})\left[\widetilde{v}_{i}(\mathbf{k}) D_{j j} k_{j} t+\widetilde{v}_{j}(\mathbf{k}) D_{i i} k_{i} t\right] .
\end{aligned}
$$

where $\imath$ is the imaginary unit. Explicit result (in second order perturbation theory in the velocity fluctuations) for the dispersion variance is provided 
below

$$
\begin{aligned}
\overline{\delta \mathcal{D}_{i j}(t)^{2}} & =\int_{k} g_{0}(\mathbf{k}, t \mid \mathbf{0}) g_{0}(-\mathbf{k}, t \mid \mathbf{0}) \widetilde{\rho}(\mathbf{k}) \mathcal{A}(\mathbf{k}) \widetilde{\rho}(-\mathbf{k}) \mathcal{A}(-\mathbf{k}) \\
& \times\left[\widetilde{C}_{i i}(\mathbf{k}) D_{j j}^{2} k_{j}^{2} t^{2}+\widetilde{C}_{j j}(\mathbf{k}) D_{i i}^{2} k_{i}^{2} t^{2}+2 \widetilde{C}_{i j}(\mathbf{k}) D_{i i} k_{i} D_{j j} k_{j} t^{2}\right] .
\end{aligned}
$$

where $A(\mathbf{k})=1-\widetilde{\mathcal{F}}(\mathbf{k})$ and $\widetilde{\mathcal{F}}(\mathbf{k})$ is the Fourier transform of the filter function (see section 2.2) and $\widetilde{C}_{i j}$ is defined in equation (10). Details related to the derivation of the unfiltered dispersion variance can be found in Dentz and de Barros [36]. As opposed to the analysis of [36], equation (40) incorporates the effect of the blockscale $\lambda$ on the sample-to-sample fluctuations of the dispersion behavior. Furthermore, contrary to the blockscale dispersion variance derived in de Barros and Rubin [35], equation (40) accounts for the effects of local scale dispersion.

\subsection{Analytical and Semi-Analytical Solutions}

In the following, analytical and semi-analytical expressions for the average and variance dispersion coefficients are developed. These expressions allows to obtain important physical insight of the temporal evolution of the dispersion coefficients and its self-averaging behavior. Without loss of generality, we employ the square filter (in Fourier space)

$$
\widetilde{\mathcal{F}}(\mathbf{k})= \begin{cases}1 & \text { for }\left|k_{i}\right| \leq \frac{\pi}{\lambda_{i}} \\ 0 & \text { otherwise }\end{cases}
$$

where $i=1, \ldots, d$, and $\lambda_{i}$ is the block size in the $\mathrm{i}$-th direction. The filter

$\widetilde{\mathcal{F}}(\mathbf{k})$ has been used to investigate the mean blockscale dispersion behavior [11, 13, 14, 15, 17] and the variance and probability density function of the blockscale dispersion tensor within a purely advective stratified random flow field [35]. The $Y=\ln K$ covariance model selected for our analysis is the Gaussian model

$$
C_{Y}\left(\mathbf{x}-\mathbf{x}^{\prime}\right)=\sigma_{Y}^{2} \prod_{i=1}^{d} \exp \left[-\frac{\left\|\mathbf{x}-\mathbf{x}^{\prime}\right\|^{2}}{2 l_{i}^{2}}\right],
$$

where $\|c d o t\|$ is the vector norm. Other correlation models for $Y$ can also be employed. Furthermore, without loss of generality, we employ a Gaussian 
distribution for the initial condition $\rho(\mathbf{x})$ with characteristic dimensions $L_{i}$ (with $i=1, \ldots, d)$ :

$$
\rho(\mathbf{x})=\prod_{i=1}^{d} \frac{1}{\sqrt{2 \pi L_{i}^{2}}} \exp \left[-\frac{x_{i}^{2}}{2 L_{i}^{2}}\right]
$$

Equation (43) allows to obtain analytical solution for the blockscale dispersion coefficients and reduces to a point-like injection in the limit $L_{i} \rightarrow 0$. Other types of source zone distributions can be adopted and the computation of the blockscale dispersion coefficients might require numerical integrations. The quantities above, namely (42) and (43), are expressed in Fourier space as

$$
\begin{aligned}
\widetilde{C}_{Y}(\mathbf{k}) & =\sigma_{Y}^{2}(2 \pi)^{d / 2} \prod_{i=1}^{d} l_{i} \exp \left[-\frac{1}{2}\left(k_{i}^{2} l_{i}^{2}\right)\right], \\
\widetilde{\rho}(\mathbf{k}) & =\prod_{i=1}^{3} \exp \left[-\frac{k_{i}^{2} L_{i}^{2}}{2}\right] .
\end{aligned}
$$

\subsubsection{Average Dispersion Coefficients}

Closed-form expressions for the ensemble and effective dispersion coefficients are presented in the limit of large Péclet, $P e \gg 1$, and $t \gg \tau_{v}$. The Péclet number is defined as $P e \equiv \tau_{D_{1}} / \tau_{v}$ where $\tau_{v}$ is the advection time scale defined by $\tau_{v}=l_{1} / \bar{v}$ (i.e. characteristic advection time over a longitudinal correlation length $l_{1}$ ) and $\tau_{D_{i}}$ the dispersion time scale given by $\tau_{D_{i}}=l_{i}^{2} / D_{i}$.

The off-diagonal elements of the average dispersion coefficients are zero for symmetry reasons. Furthermore, in first-order perturbation theory in the fluctuation variance of $Y$, only the longitudinal dispersion coefficients are of macroscopic order, i.e., their leading order is independent of the local scale dispersion coefficients. Thus, we focus here only on the longitudinal dispersion coefficients. Substituting (41), (44) and (45) in (37), we obtain the ensemble dispersion coefficient

$$
D_{11}^{e n s}(t \rightarrow \infty) \equiv D_{11}^{e n s, \infty}=D_{11}+\sqrt{\frac{\pi}{2}} \sigma_{Y}^{2} \bar{v} l_{1}\left[1-\prod_{i=2}^{d} \operatorname{erf}\left(\frac{\pi}{\sqrt{2} \lambda_{i}}\right)\right]
$$


which is similar to the asymptotic expressions derived in Rubin et al. [11, 13]. For the effective dispersion coefficient we obtain in analogy

$$
D_{11}^{e f f}(t)=D_{11}^{e n s, \infty}-\sqrt{\frac{\pi}{2}} \sigma_{Y}^{2} \bar{v} l_{1} \frac{1-\prod_{i=2}^{d} \operatorname{erf}\left[\frac{\pi\left(1+\frac{4 t}{\tau_{D_{i}}}\right)}{\sqrt{2} \lambda_{i}}\right]}{\prod_{i=2}^{d}\left(1+\frac{4 t}{\tau_{D_{i}}}\right)^{1 / 2}} .
$$

\subsubsection{Dispersion Variance for a Fully Isotropic Case}

The dispersion variance, equation (40), can evaluated through numerical quadratures. However, a semi-analytical expression can be obtained by considering a fully isotropic case: an isotropic source $\left(L=L_{i}\right)$, a statistically isotropic $Y$-field $\left(l \equiv l_{i}\right)$, an isotropic local scale dispersion $\left(D_{i}=D\right)$ and a cubic block of dimensions $\lambda$. The semi-analytical solution is obtained by making use of the filter (41), the correlation function (42) and the inlet distribution (43). We report the results for a three-dimensional flow field $(d=3)$. The dispersion variance that complies with the aforementioned simplifying assumptions is

$$
\overline{\delta \mathcal{D}_{i i}(t)^{2}}=\frac{8}{35} \sigma_{Y}^{2} l^{2} \bar{v}^{2} \frac{\left[(L / l)^{2}+\frac{2 t}{\tau_{D}}\right]^{2}}{\left[1+2(L / l)^{2}+\frac{4 t}{\tau_{D}}\right]^{5 / 2}}-\frac{4 D^{2} t^{2} \gamma}{(2 \pi)^{3}} \int_{\mu=0}^{\infty} \Omega(t, \mu) d \mu
$$

where $\gamma=\bar{v} \sigma_{Y}^{2} l^{3}(2 \pi)^{3 / 2}$ and $\Omega(t, \mu)$ is:

$$
\begin{aligned}
\Omega(t, \mu)= & -\frac{4 \sqrt{2} \pi \mu}{[\lambda \Phi(t, \mu)]^{5}} \exp \left[-\frac{3}{2} \frac{\pi^{2}}{\lambda^{2}} \Phi(t, \mu)\right] \Psi_{1}(t, \mu) \times \\
& {\left[\Psi_{2}(t, \mu)+\Psi_{3}(t, \mu)-\Psi_{4}(t, \mu)\right] }
\end{aligned}
$$

with $\Phi, \Psi_{1}, \Psi_{2}, \Psi_{3}$ and $\Psi_{4}$ defined as

$$
\Phi(t, \mu)=2\left(\frac{2 l^{2} t}{\tau_{D}}+\mu+L^{2}\right)+l^{2}
$$




$$
\begin{aligned}
& \Psi_{1}(t, \mu)=\lambda \sqrt{2 \pi \Phi(t, \mu)} \operatorname{erf}\left[\frac{\pi \sqrt{\Phi(t, \mu)}}{\lambda \sqrt{2}}\right] \exp \left[\frac{\pi^{2} \Phi(t, \mu)}{2 \lambda^{2}}\right]-2 \pi \Phi(t, \mu) \\
& \Psi_{2}(t, \mu)=\lambda^{3} \sqrt{\Phi(t, \mu) \pi} \exp \left[\frac{\pi^{2} \Phi(t, \mu)}{2 \lambda^{2}}\right] \operatorname{erf}\left[\frac{\pi}{\lambda} \sqrt{\frac{\Phi(t, \mu)}{2}}\right] \\
& \Psi_{3}(t, \mu)=\lambda \sqrt{\Phi(t, \mu) \pi}\left(2 \pi^{2} \Phi(t, \mu)+4 \lambda^{2}\right) \exp \left[\frac{\pi^{2} \Phi(t, \mu)}{2 \lambda^{2}}\right] \operatorname{erf}\left[\frac{\pi}{\lambda} \sqrt{\frac{\Phi(t, \mu)}{2}}\right] \\
& \Psi_{4}(t, \mu)=2 \sqrt{2} \lambda^{4} \exp \left[\frac{\pi^{2} \Phi(t, \mu)}{\lambda^{2}}\right] \operatorname{erf}\left[\frac{\pi}{\lambda} \sqrt{\frac{\Phi(t, \mu)}{2}}\right]^{2}+\pi \sqrt{2} \lambda^{2} \Phi(t, \mu)
\end{aligned}
$$

Summarizing, equation (48) contains one integral that needs to be evaluated numerically. Notice that the following limiting cases hold for equation (48):

$$
\begin{aligned}
\lim _{\lambda \rightarrow \infty} \overline{\delta \mathcal{D}_{i i}(t)^{2}} & =\frac{8}{35} \sigma_{Y}^{2} l^{2} \bar{v}^{2} \frac{\left[(L / l)^{2}+\frac{2 t}{\tau_{D}}\right]^{2}}{\left[1+2(L / l)^{2}+\frac{4 t}{\tau_{D}}\right]^{5 / 2}} \\
\lim _{\lambda \rightarrow 0} \overline{\delta \mathcal{D}_{i i}(t)^{2}} & =0
\end{aligned}
$$

When the block scale is large compared to the heterogeneity correlation scale, the blockscale dispersion variance tends to the fully upscaled dispersion variance reported in Dentz and de Barros [36] whereas for a very small block scale (e.g. fine numerical grid), all the heterogeneity is captured in the velocity field and the dispersion variance tends zero since the dispersive fluxes are small. These limiting cases will be shown in the upcoming section.

\section{Results and Analysis}

In this section we analyze the joint impact of the blockscale, source injection scale, local scale dispersion and the statistical anisotropy ratio in the overall mean effective dispersion behavior and its fluctuations. In all simulations, we set $\bar{v}=1, \sigma_{Y}^{2}=1, l_{i}=1$ and $\lambda=\lambda_{i}$ (for $i=1,2,3$ ) unless stated otherwise. Note that $l_{i}$ corresponds to the correlation scale of $Y$ and 
not the integral scale as adopted in several other works [e.g. 21, 11]. For a correspondence between the integral scale and the correlation scale for the Gaussian covariance model (44), see chapter 2 of Rubin [6]. For the purpose of illustration, the local scale dispersion tensor is set to be isotropic and components equal to $D$.

Figure 3 displays the average effective dispersion coefficient (47). Larger block sizes lead to larger effective dispersion values. These results are expected and are in agreement with previous analysis [11, 13, 35]. Larger values of $\lambda$ implies that a larger portion of the spatially variable flow field is homogenized. Figure 3 also illustrates the temporal evolution of the effective blockscale dispersion coefficient $D_{11}^{e f f}(t)$. It evolves roughly on the dispersion time scale $\tau_{D}$. For decreasing ratio between block and correlation scales, it evolves faster because the characteristic heterogeneity scales that contribute to solute dispersion are below the correlation scale and thus are sampled faster by the solute plume (see also discussion in [13]). Notice that the ensemble blockscale dispersion coefficient (46) evolves towards its asymptotic value on the advection scale $\tau_{v}$. Thus, as for its unfiltered counterpart [25, 26, 31, 32], at preasymptotic times, the ensemble blockscale dispersion coefficients quantify artificial sample to sample fluctuations of the center of mass position of the coarse-grained plume. They overestimate actual solute dispersion due to subscale velocity fluctuation. The effective blockscale dispersion coefficients on the other hand reflect the dispersion effect due to local scale dispersion and subscale velocity fluctuations only.

Figure 4 depicts the temporal evolution of the blockscale dispersion variance for a point source injection in a fully isotropic scenario with $P e=10^{3}$ where $P e \equiv \bar{v} l / D$. Notice that the dispersion variance is given by $\sigma_{D i i}^{2} \equiv$ $\overline{\delta \mathcal{D}_{i i}(t)^{2}}$ in Figure 4. The results were obtained using the semi-analytical expression given by equation (48). As expected, the dispersion variance decreases with decreasing block size $\lambda$. For larger $\lambda$ values, more subscale variability is homogenized since less heterogeneity is captured on the blockscale (such as in a coarse numerical grid). Therefore, this wiped out variability needs to be compensated in the dispersion tensor (see figure 31). For large $\lambda$, the longitudinal blockscale dispersion variance (48) approaches the fully upscaled value derived in [36], see the limit in equation (52). Figure 4 also shows that the values obtained for the blockscale dispersion variance are small hence indicating that the uncertainty is not large. From a practical point of view, this result illustrates the potential of the blockscale, which is in general defined by field sampling campaigns [11], in reducing the uncertainty 


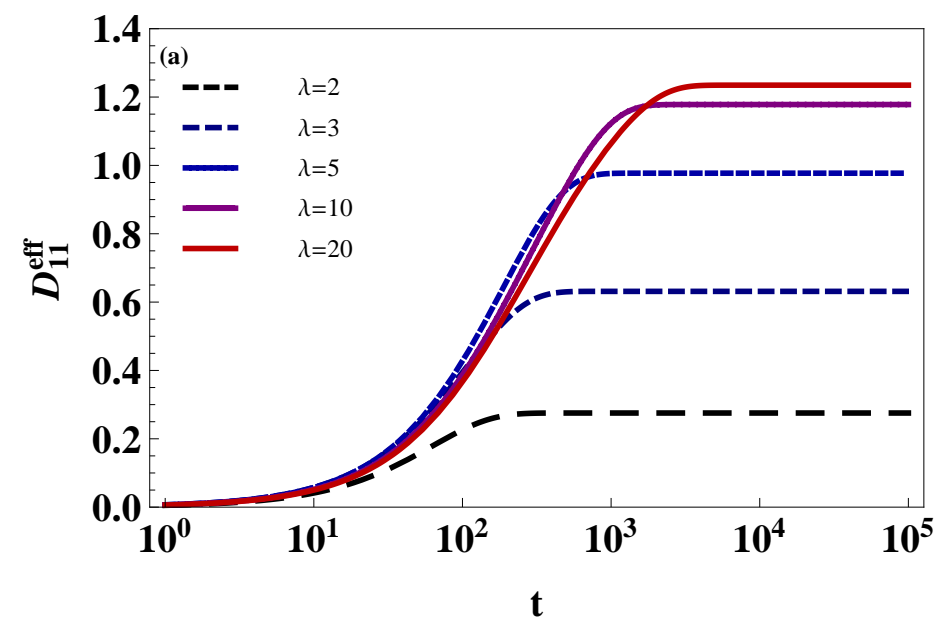

Figure 3: Temporal evolution of the average effective longitudinal blockscale dispersion coefficient as a function of $\lambda$. Analysis performed for a fully isotropic case and $P e=10^{3}$.

in transport predictions.

The other remarkable observation in figure 4 is that the sample-to-sample fluctuations of the dispersion tends to zero for large times. Therefore, dispersion is self-averaging. However, by comparing the curve for $\lambda \rightarrow \infty$ with the result obtained for $\lambda=2$, we observe that the self-averaging behavior is anticipated for smaller block sizes. For the fully upscaled dispersion tensor, i.e. $\lambda \rightarrow \infty$, the system self-averages approximately at time $t \approx 10^{7}$ while for $\lambda=2$, the system self-averages around the diffusive time scale, i.e. $t \approx 10^{3}$ This result has implications in field applications since, in general, numerical grid design are dictated by a coarse hydraulic conductivity measurement network. In other words, our results indicate the significance of blockscale in reducing the variance of the large scale plume spreading behavior. As shown in figure 4, conditioning model predictions on conductivity measurements and other types of information has the strong potential of reduce both the uncertainty of the large scale dispersion behavior and the self-averaging characteristic time.

Another key parameter in reducing the dispersion variance and controlling the self-averaging rate of the spreading behavior of the plume is the local scale dispersion coefficient $D$. Local-scale dispersion tends to smooth out concentration gradients thus diminishing the differences observed from the 


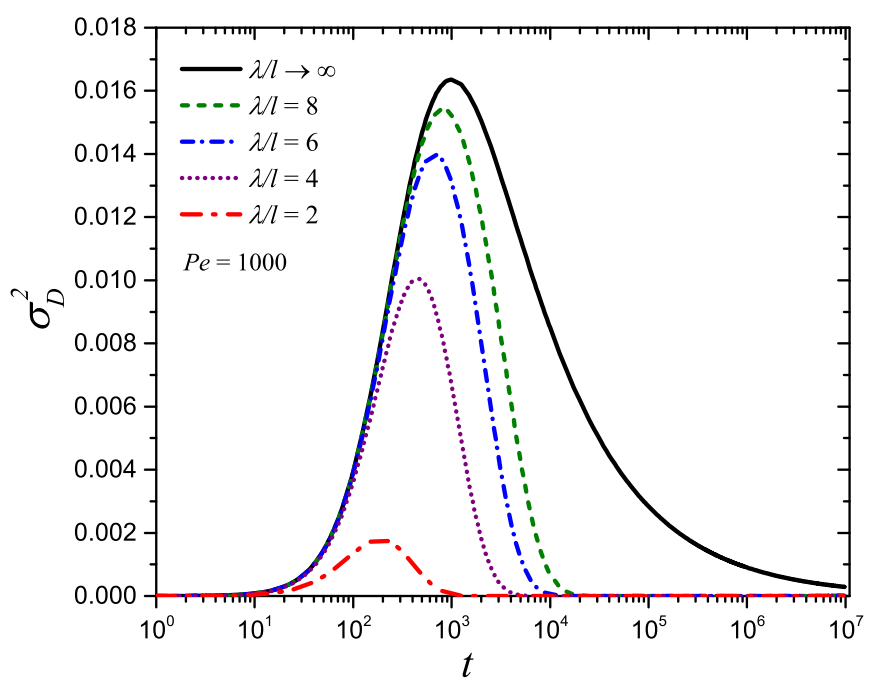

Figure 4: Temporal evolution of the dispersion variance as a function of the block scale $\lambda$. Results obtained for a fully isotropic case, point source injection and $P e=10^{3}$.

ensemble. Figure 5 illustrates the dependency of the peak dispersion variance time on the block size for different $P e$. The time of the peak dispersion variance is denoted by $t_{p}$ and mathematically defined as

$$
t_{p}(\lambda)=\arg \max _{t \in[0, \infty)} \sigma_{D 11}^{2}(t ; \lambda) .
$$

As shown in figure 5, the time of peak dispersion variance, $t_{p}$, will depend on $\lambda$. The time of peak $t_{p}$ is more sensitive to $\lambda$ for higher $P e$, e.g. advection dominated scenarios. For lower $P e, t_{p}$ reaches its asymptotic value for smaller block sizes. Close inspection of figure 5 shows that the asymptotic value for $t_{p}$ is equal to the diffusive characteristic time $\tau_{D}=l^{2} / D_{d}$. For $P e=10^{3}$, the asymptotic value for $t_{p}$ is reached around $\lambda \approx 25 l$ whereas for $P e=10^{2}, t_{p}$ approaches its asymptote at $\lambda \approx 10 l$.

Next, we investigate the impact of the source dimension $L$ in reducing the blockscale dispersion variance. The injection zone is characterized by an extended transverse line source. Figure [6 shows the time evolution of the dispersion variance for the following characteristic transverse length scale of 


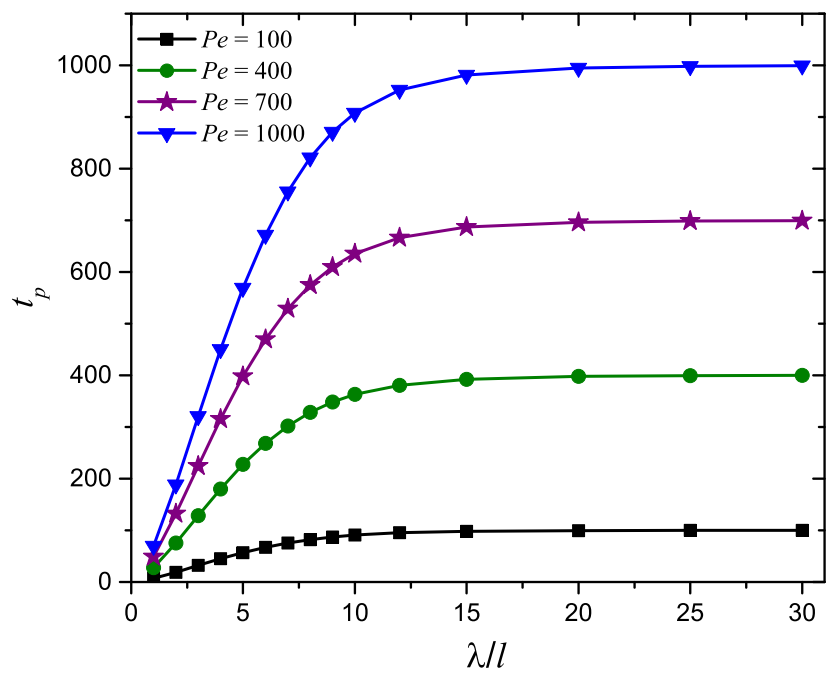

Figure 5: Time of the peak dispersion variance $\left(t_{p}\right)$ versus the dimensionless block scale $\lambda / l$. Results obtained for a fully isotropic case, point source injection, $\bar{u}=1, \sigma_{Y}^{2}=1$, $l=1$ and for different $P e$.

the source distribution: $L_{2}=2 l$ and $L_{2}=10 l$ for a fixed $L_{1}=L_{3}=0.1 l$. As depicted in figure 6 , the sample-to-sample fluctuations of the blockscale dispersion coefficient reduces with increasing $L_{2}$. This effect can be observed by comparing figures [6. a with [6.b. For large injection sources, the solute plume approaches ergodicity and transport is less subject to uncertainty [27, 29, 35]. The impact of $L$ on the uncertainty of spreading was also investigated in detail in the literature [59, 54, 36].

The uncertainty of the large scale dispersion also depends on the statistical anisotropy ratio $f_{R}$ of the log-conductivity. The anisotropy ratio is defined as $f_{R}=l_{3} / l_{h}$, with $l_{1}=l_{2} \equiv l_{h}$ denoting the horizontal correlation scale and $l_{3}$ is the vertical correlation scale of $Y$. For many geological sites, values of $f_{R}$ are typically $f_{R} \ll 1[6]$. Figure 7 displays the impact of $f_{R}$ in reducing the blockscale dispersion variance. The dispersion variance results are obtained for $f_{R}=0.1$ and 1 . For a smaller anisotropy ratio, see curves for $f_{R}=0.1$, dilution is augmented since a lower value of $f_{R}$ tends to generate solute plumes with fingers of vertical characteristic scale $l_{3}$. These thin solute fingers are smoothed out by $D$ thus destroying the variability of the 

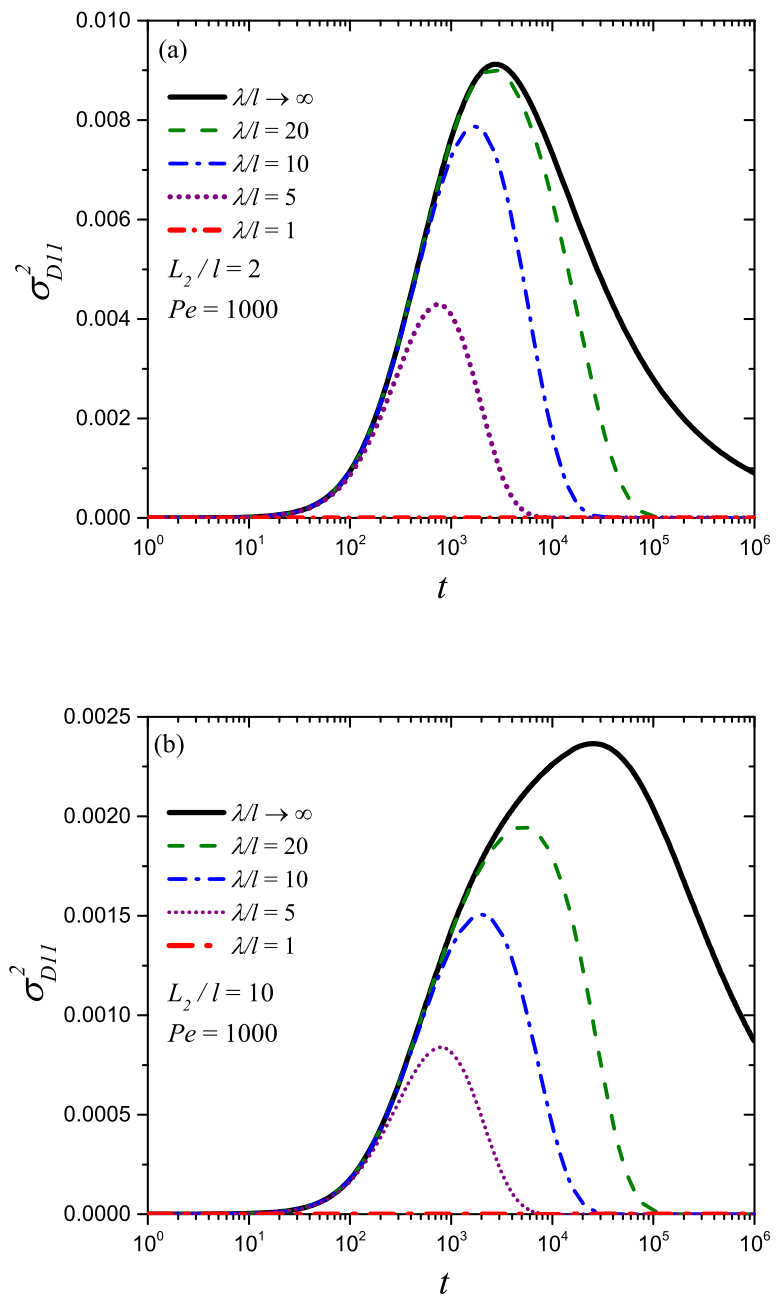

Figure 6: Temporal evolution of the dispersion variance as a function of the block scale $\lambda$ for different transverse source extension $L_{2} / l=[2,10]$ with fixed $L_{1}=L_{3}=0.1$. Results obtained for a fully isotropic case and $P e=10^{3}$. 


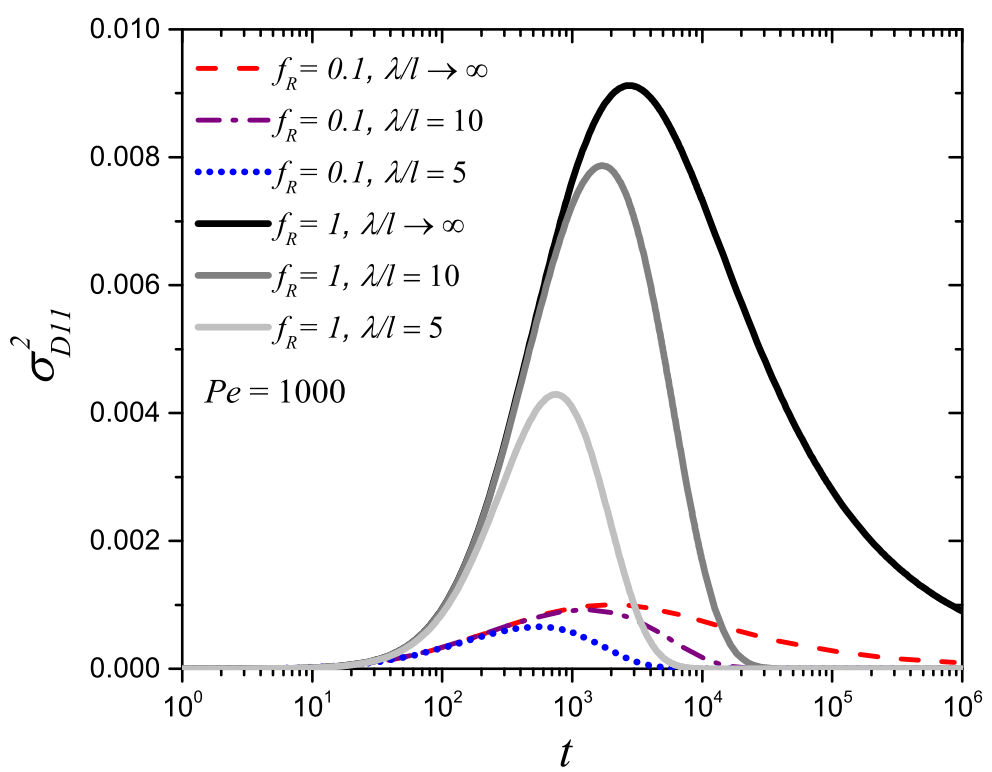

Figure 7: Temporal evolution of the longitudinal dispersion variance as a function of the block scale $\lambda$ and the statistical anisotropy ratio $f_{R}$. Results for $L_{1}=L_{3}=0.1 l_{h}, L_{2}=2 l_{h}$, $P e=10^{3}, f_{R}=l_{3} / l_{h}$ where $l_{h} \equiv l_{1}=l_{2}$. Parameter values used $\bar{u}=1, \sigma_{Y}^{2}=1$ and $l_{h}=1$.

concentration distribution in a single realization [60, 61].

\section{Summary and Conclusions}

Numerical models tend to use coarse numerical grid blocks for two main reasons. The first one is that the hydraulic conductivity and flow data are, in general, obtained over a coarse measurement grid and therefore the flow field is partially resolved. Second, stochastic modeling of transport in complex hydro-systems often calls for brute force numerical Monte Carlo simulations with a fine numerical mesh such that solute spreading and mixing are accurately predicted. This leads to a computational burden which can be alleviated by using coarse numerical grid blocks. For such reasons, it is important to wisely allocate computational resources [41, 62]. A possible strategy consists in accounting for the effects of flow variability at the subgrid scale in dispersion coefficients. Two fundamental issues are pursued in 
this article. We discuss two distinct blockscale dispersion concepts, which represent different aspects of subscale velocity fluctuation, and quantify the uncertainty in terms of the variance of the sample to sample variance of the single realization blockscale dispersion coefficients.

We discuss the concepts of ensemble and effective dispersion for the quantification of subscale velocity fluctuations. As for its unfiltered counterpart, effective dispersion reflects the dispersion effect due to velocity fluctuations in a single aquifer realization. The ensemble dispersion coefficient quantifies an artificial dispersion effect due to sample to sample velocity fluctuations between aquifer realizations. Thus, the effective blockscale dispersion concept is better suited for the quantification of dilution and chemical reactions within a coarse-grained transport framework than the ensemble dispersion concepts. Based on these blockscale dispersion conceptualizations, we propose two stochastic surrogate models from which we derive the governing equations for the respective coarse-grained concentration distributions.

The uncertainty of the blockscale dispersion tensor is only well-defined for the effective blockscale dispersion concepts because they are derived by the ensemble average over their single aquifer realization counterpart. The ensemble dispersion coefficient is genuinely an ensemble concept and defined in terms of the ensemble of aquifer realizations as a whole. Uncertainty of the blockscale dispersion coefficients is manifested through their variance, which strongly depends on the initial dimensions of the solute plume and the scale of the homogenization block.

We develop explicit (semi-) analytical solutions for the effective blockscale dispersion coefficients and the blockscale dispersion variance. Notice that our analytical and semi-analytical results are limited to uniform-in-the-mean flow fields, unbounded domains, low-to-mild heterogeneity $\left(\sigma_{Y}^{2}<1\right)$, a Gaussian covariance function and an instantaneous injection of a non-reactive solute following a spatial Gaussian distribution. As shown in our results, the interaction between local scale dispersion and the scale of the block is important in determining the time of peak uncertainty in the dispersion tensor. The impact of the smaller block sizes in diminishing the uncertainty in the overall dispersion tensor is related to the fact that ergodicity is achieved, with respect to the subgrid correlation scale, rather quickly $\left(L_{j} \gg l_{j \lambda}\right.$ for $j=2,3$ with $l_{j \lambda}$ denoting the subscale correlation length). Furthermore, the dispersion behavior self-averages in 3D flow fields. We also show that under finite $P e$ conditions, lower values in the statistical anisotropy ratio of the log-conductivity contribute to reduce the variability in the sample-to-sample 
fluctuations of the large scale dispersion behavior. This implies that dispersion self-averages faster for lower $f_{R}$ (which is typically the conditions found in field sites, i.e. $\left.f_{R} \ll 1\right)$. As expected, the initial size of the solute plume is critical in determining the overall evolution of the dispersion variance: For increasing transverse dimensions of the solute plume, thus approaching ergodicity, the variance of dispersion decreases tending to zero. Aside from the implications associated with computation time reduction, the results shown in this work highlights the potential of the blockscale in decreasing the uncertainty of the effective transport behavior of the plume. The reduction of both computational time and uncertainty in dispersion coefficients are particularly important in the context of probabilistic human health risk analysis where fine resolution of the numerical grid and large number of Monte Carlo simulations are required to accurately capture transport dynamics of multireactive species and estimate the probability of extreme events [63, 64]. For such reasons, blockscale dispersion concepts could provide approximations for subscale processes which can lead to a reduction in the computational costs.

The analysis performed in this work can be adapted to incorporate different release conditions (in space and time), heterogeneity correlation model and reactive transport at the cost of performing additional numerical integrations. As an outlook to the future, a critical issue not covered in this work is in determining the rate in which dispersion coefficients self-average for a disordered medium with higher levels of heterogeneity and in the presence of sinks/sources (e.g. pumping well). Challenges remain when addressing transport in geological formations strongly heterogeneous permeability fields. High contrasts of the conductivity lead to early breakthrough and late arrivals and a different upscaling methodology might be required. For the case of high heterogeneity, alternative upscaling approaches, such as the one presented in Fernàndez et al. [65] and Li et al. [66] are available. It is worth mentioning that the applicability of perturbation methods can be extended to fields with higher heterogeneity by using the approach documented in Neuman [67]. Our analysis was limited to blocks of identical dimensions (i.e. $\lambda_{1}=\lambda_{2}=\lambda_{3}=\lambda$ ). However in many applications, numerical meshes are designed adaptively and therefore homogenized regions will have different sizes. In this case, each numerical block would be characterized with a distinct dispersion coefficient. The work of Dentz and de Barros [50] illustrates how the current framework can be used with a spatiotemporal dynamic filtering scale $\lambda_{i}$. 
A detailed discussion on the application of blockscale dispersion concept is provided in ch. 10, p. 253 of Rubin [6] and Bellin et al. [68]. The approach proposed by Rubin and co-workers $[11,13,6$ ] consists of solving flow and transport over a randomly generated coarse hydraulic conductivity field. Transport on the coarse field is solved using the assigned average blockscale dispersion coefficients [68] which will depend on the dimensions on the grid block. The assigned average blockscale dispersion value is estimated from perturbation theory. In addition to the average blockscale dispersion, we provide expressions for the associated uncertainty epitomized by its variance. Hence, we expand the pioneering works of Rubin and collaborators [11, 13] by providing expressions for the uncertainty in the blockscale dispersion tensor while accounting for $P e$, plume scale and $f_{R}$. The semi-analytical expressions for the blockscale dispersion variance provided in de Barros and Rubin [35] are valid for infinite $P e$ and $f_{R} \ll 1$. Our approach provides the low-order statistical moments of the blockscale dispersion tensor and allows to randomly assign dispersion coefficients if its probability density function (PDF) is available. As shown in de Barros and Rubin [35], the blockscale PDF can be obtained by evoking entropy principles with limited information such as the upper/lower bounds of the random variable and low order statistics, namely the mean and variance.

The results of this work provide a quantitative basis for the use of dispersion coefficients in coarse-grained transport models and numerical simulations as they shed new light on the physical meaning of different blockscale dispersion concepts and its uncertainty. The temporal evolution of the blockscale dispersion variance provides information relevant to the efficiency in solute mixing as well as on ergodicity of the transport process. The uncertainty analysis of the blockscale dispersion coefficient performed in this work supports the conclusions of Rubin et al. [13] in which plume ergodicity is achieved with respect to the blockscale. Our findings may be relevant for the interpretation of dispersion data from field and laboratory experiments and how they compare with model predictions using numerical models. The concept of effective blockscale dispersion and the associated surrogate Lagrangian and Eulerian transport models may provide a suitable frame for the the quantification of mixing and chemical reactions in a coarse-grained transport framework. 


\section{Acknowledgements}

The first author acknowledges Yoram Rubin for fruitful discussions on the topic of block-effective macrodispersion at the early stages of this work. The second author acknowledges the support of the European Research Council (ERC) through the project MHetScale (contract number 617511). We thank all three reviewers for their constructive comments.

\section{Appendix A. Perturbation Expressions}

Using (41), (44) and (45) in (37) and (38), we find that the longitudinal ensemble and effective dispersion coefficients can be written in the limit of $P e \gg 1$ and $t \gg \tau_{v}$ as

$$
\begin{aligned}
D_{11}^{e n s}(t) & =D_{11}+\sqrt{\frac{\pi}{2}} \sigma_{Y}^{2} \bar{v} l_{1} M(1, \ldots, 1), \\
D_{11}^{e f f}(t) & =D_{11}+\sqrt{\frac{\pi}{2}} \sigma_{Y}^{2} \bar{v} l_{1}[M(1, \ldots, 1) \\
& \left.-M\left(1, \sqrt{1+2 L_{2}^{2} / l_{2}^{2}+4 t / \tau_{D_{2}}}, \ldots, \sqrt{1+2 L_{d}^{2} / l_{d}^{2}+4 t / \tau_{D_{d}}}\right)\right],
\end{aligned}
$$

where the auxiliary function $M(\mathbf{A})$ is given by

$$
\begin{aligned}
& M(\mathbf{A})=\sqrt{\frac{2}{\pi}} \int_{0}^{\infty} d t^{\prime}\left[\int_{k} \exp \left(-\frac{k_{i}^{2} A_{i}^{2}}{2}-i k_{1} t^{\prime}\right)-\right. \\
& \left.\prod_{i=1}^{d} \int_{-\pi / \lambda_{i}}^{\pi / \lambda_{i}} \frac{d k_{i}}{2 \pi} \exp \left(-\frac{k_{i}^{2} A_{i}^{2}}{2}-i k_{1} t^{\prime}\right)\right]+\ldots
\end{aligned}
$$

where the dots denote terms of order $P e^{-1}$ and $\tau_{v} / t$. We note that

$$
\int_{0}^{\infty} d t^{\prime} \exp \left(-i k_{1} t^{\prime}\right)=\pi \delta\left(k_{1}\right)
$$

Thus, we obtain for $M(\mathbf{A})$

$$
M(\mathbf{A})=M\left(1, A_{2}, \ldots, A_{d}\right)=\frac{1-\prod_{i=2}^{d} \operatorname{erf}\left(\frac{\pi}{\sqrt{2} \lambda_{i} \sqrt{A_{i}}}\right)}{\prod_{i=2}^{d} \sqrt{A_{i}}} .
$$




\section{References}

[1] L. Gelhar, C. Axness, Three-dimensional stochastic analysis of macrodispersion in aquifers, Water Resour. Res. 19 (1) (1983) 161-180.

[2] G. Dagan, Time-dependent macrodispersion for solute transport in anisotropic heterogeneous aquifers, Water Resour. Res. 24 (9) (1988) $1491-1500$.

[3] A. Fiori, I. Janković, G. Dagan, Flow and transport in highly heterogeneous formations: 2. semianalytical results for isotropic media, Water Resour. Res. 39 (9).

[4] T. Le Borgne, M. Dentz, D. Bolster, J. Carrera, J.-R. De Dreuzy, P. Davy, Non-fickian mixing: Temporal evolution of the scalar dissipation rate in heterogeneous porous media, Adv. Water Resour. 33 (12) (2010) 1468-1475.

[5] M. Dentz, T. Le Borgne, A. Englert, B. Bijeljic, Mixing, spreading and reaction in heterogeneous media: A brief review, J. Contam. Hydrol. 120 (2011) 1-17.

[6] Y. Rubin, Applied stochastic hydrology, Oxford University Press, Oxford, 2003.

[7] G. Dagan, Upscaling of dispersion coefficients in transport through heterogeneous formations, Computational Methods in Water Resources X 1 (1994) 431-439.

[8] R. Beckie, Measurement scale, network sampling scale, and groundwater model parameters, Water Resour. Res. 32 (1) (1996) 65-76.

[9] R. Beckie, A. A. Aldama, E. F. Wood, Modeling the large-scale dynamics of saturated groundwater flow using spatial-filtering theory: 1 . theoretical development, Water Resour. Res. 32 (5) (1996) 1269-1280.

[10] A. R. Mehrabi, M. Sahimi, Coarsening of heterogeneous media: application of wavelets, Phys. Rev. Lett. 79 (22) (1997) 4385.

[11] Y. Rubin, A. Sun, R. Maxwell, A. Bellin, The concept of block-effective macrodispersivity and a unified approach for grid-scale-and plume-scaledependent transport, J. Fluid Mech. 395 (1999) 161-180. 
[12] S. Attinger, Generalized coarse graining procedures for flow in porous media, Comput. Geosci. 7 (4) (2003) 253-273.

[13] Y. Rubin, A. Bellin, A. E. Lawrence, On the use of block-effective macrodispersion for numerical simulations of transport in heterogeneous formations, Water Resour. Res. 39 (9).

[14] A. Bellin, A. E. Lawrence, Y. Rubin, Models of sub-grid variability in numerical simulations of solute transport in heterogeneous porous formations: three-dimensional flow and effect of pore-scale dispersion, Stoch. Env. Res. Risk A 18 (1) (2004) 31-38.

[15] J. Eberhard, Upscaling for stationary transport in heterogeneous porous media, Multiscale Model Sim. 3 (4) (2005) 957-976.

[16] D. Fernàndez-Garcia, J. Gómez-Hernández, Impact of upscaling on solute transport: traveltimes, scale dependence of dispersivity, and propagation of uncertainty, Water Resour. Res. 43 (2).

[17] A. E. Lawrence, Y. Rubin, Block-effective macrodispersion for numerical simulations of sorbing solute transport in heterogeneous porous formations, Adv. Water Resour. 30 (5) (2007) 1272-1285.

[18] J. Cortinez, A. Valocchi, P. Herrera, Effective grid-dependent dispersion coefficient for conservative and reactive transport simulations in heterogeneous porous media, in: AGU Fall Meeting Abstracts, Vol. 1, 2013, p. 1105 .

[19] J. Wang, P. K. Kitanidis, Analysis of macrodispersion through volume averaging: comparison with stochastic theory, Stoch. Env. Res. Risk A. 13 (1-2) (1999) 66-84.

[20] B. D. Wood, F. Cherblanc, M. Quintard, S. Whitaker, Volume averaging for determining the effective dispersion tensor: Closure using periodic unit cells and comparison with ensemble averaging, Water Resour. Res. $39(8)$.

[21] G. Dagan, Solute transport in heterogeneous porous formations, J. Fluid Mech. 145 (1984) 151-77. 
[22] S. Neuman, C. Winter, C. Newman, Stochastic theory of field-scale fickian dispersion in anisotropic porous media, Water Resour. Res. 23 (3) (1987) 453-466.

[23] H. Quinodoz, A. Valocchi, Stochastic analysis of the transport of kinetically sorbing solutes in aquifers with randomly heterogeneous hydraulic conductivity, Water Resour. Res.h 29 (9) (1993) 3227-3240.

[24] K.-C. Hsu, D. Zhang, S. Neuman, Higher-order effects on flow and transport in randomly heterogeneous porous media, Water Resour. Res. 32 (3) (1996) 571-582.

[25] P. K. Kitanidis, Prediction by the method of moments of transport in a heterogeneous formation, J. Hydrol. 102 (1) (1988) 453-473.

[26] G. Dagan, Transport in heterogeneous porous formations: Spatial moments, ergodicity, and effective dispersion, Water Resour. Res. 26 (6) (1990) 1281-1290.

[27] G. Dagan, Dispersion of a passive solute in non-ergodic transport by steady velocity fields in heterogeneous formations, J. Fluid Mech 233 (1) (1991) 99 .

[28] H. Rajaram, L. W. Gelhar, Plume scale-dependent dispersion in heterogeneous aquifers: 2. eulerian analysis and three-dimensional aquifers, Water Resour. Res. 29 (9) (1993) 3261-3276.

[29] A. Fiori, On the influence of pore-scale dispersion in nonergodic transport in heterogeneous formations, Transport Porous Med. 30 (1) (1998) $57-73$.

[30] Y.-K. Zhang, V. di Federico, Solute transport in three-dimensional heterogeneous media with a gaussian covariance of log hydraulic conductivity, Water Resour. Res. 34 (8) (1998) 1929-1934.

[31] M. Dentz, H. Kinzelbach, S. Attinger, W. Kinzelbach, Temporal behavior of a solute cloud in a heterogeneous porous medium: 1. point-like injection, Water Resour. Res. 36 (12) (2000) 3591-3604.

[32] M. Dentz, H. Kinzelbach, S. Attinger, W. Kinzelbach, Temporal behavior of a solute cloud in a heterogeneous porous medium: 2. spatially extended injection, Water Resour. Res. 36 (12) (2000) 3605-3614. 
[33] S. Attinger, M. Dentz, H. Kinzelbach, W. Kinzelbach, Temporal behaviour of a solute cloud in a chemically heterogeneous porous medium, J. Fluid Mech. 386 (1999) 77-104.

[34] D. Bolster, M. Dentz, Anomalous dispersion in chemically heterogeneous media induced by long-range disorder correlation, J. Fluid Mech. 695 (2012) 366-389.

[35] F. P. J. de Barros, Y. Rubin, Modelling of block-scale macrodispersion as a random function, J. Fluid Mech. 676 (2011) 514.

[36] M. Dentz, F. P. J. de Barros, Dispersion variance for transport in heterogeneous porous media, Water Resour. Res. 49 (6) (2013) 3443-3461.

[37] M. Clincy, H. Kinzelbach, Stratified disordered media: exact solutions for transport parameters and their self-averaging properties, J. Phys. A Math. Gen. 34 (36) (2001) 7141.

[38] N. Suciu, C. Vamoş, J. Vanderborght, H. Hardelauf, H. Vereecken, Numerical investigations on ergodicity of solute transport in heterogeneous aquifers, Water Resour. Res. 42 (4).

[39] N. Suciu, Diffusion in random velocity fields with applications to contaminant transport in groundwater, Adv. Water Resour. 69 (2014) 114133.

[40] H. Risken, Fokker-Planck Equation, Springer, 1996.

[41] P. Leube, F. P. J. de Barros, W. Nowak, R. Rajagopal, Towards optimal allocation of computer resources: Trade-offs between uncertainty quantification, discretization and model reduction, Environ. Modell. Softw. 50 (2013) 97-107.

[42] R. Ababou, D. McLaughlin, L. Gelhar, A. Tompson, Numerical simulation of three-dimensional saturated flow in randomly heterogeneous porous media, Transport in Porous Med. 4 (6) (1989) 549-565.

[43] Y. Efendiev, L. Durlofsky, S. Lee, Modeling of subgrid effects in coarsescale simulations of transport in heterogeneous porous media, Water Resour. Res. 36 (8) (2000) 2031-2041. 
[44] F. P. J. de Barros, Y. Rubin, R. Maxwell, The concept of comparative information yield curves and its application to risk-based site characterization, Water Resour. Res. 45 (6) (2009) W06401.

[45] D. M. Tartakovsky, Assessment and management of risk in subsurface hydrology: A review and perspective, Adv. Water Resour. 51 (2013) $247-260$.

[46] Y. Rubin, Stochastic modeling of macrodispersion in heterogeneous porous medias, Water Resour. Res. 26 (1).

[47] J. H. Cushman, On unifying the concepts of scale, instrumentation, and stochastics in the development of multiphase transport theory, Water Resour. Res. 20 (11) (1984) 1668-1676.

[48] P. Baveye, G. Sposito, The operational significance of the continuum hypothesis in the theory of water movement through soils and aquifers, Water Resour. Res. 20 (5) (1984) 521-530.

[49] M. Quintard, S. Whitaker, Transport in ordered and disordered porous media ii: Generalized volume averaging, Transport in Porous Med. 14 (2) (1994) 179-206.

[50] M. Dentz, F. P. J. de Barros, Mixing-scale dependent dispersion for transport in heterogeneous flows, J. Fluid Mech. 777 (2015) 178-195.

[51] A. Fiori, G. Dagan, I. Jankovic, A. Zarlenga, The plume spreading in the made transport experiment: Could it be predicted by stochastic models?, Water Resources Research 49 (5) (2013) 2497-2507.

[52] M. Dentz, T. Le Borgne, J. Carrera, Effective transport in random shear flows, Physical Review E 77 (2) (2008) 020101.

[53] A. Fiori, The lagrangian concentration approach for determining dilution in aquifer transport: Theoretical analysis and comparison with field experiments, Water Resour. Res. 37 (12) (2001) 3105-3114.

[54] A. Fiori, I. Janković, Can we determine the transverse macrodispersivity by using the method of moments?, Adv. Water Resour. 28 (6) (2005) 589-599. 
[55] O. A. Cirpka, F. P. J. de Barros, G. Chiogna, M. Rolle, W. Nowak, Stochastic flux-related analysis of transverse mixing in two-dimensional heterogeneous porous media, Water Resour. Res. 47 (6) (2011) W06515.

[56] J.-P. Bouchaud, A. Georges, Anomalous diffusion in disordered media: statistical mechanisms, models and physical applications, Phys. Rep. 195 (4) (1990) 127-293.

[57] N. Suciu, C. Vamoş, J. Eberhard, Evaluation of the first-order approximations for transport in heterogeneous media, Water Resour. Res. $42(11)$.

[58] J. Eberhard, N. Suciu, C. Vamos, On the self-averaging of dispersion for transport in quasi-periodic random media, J. Phys. A: Math Theor 40 (4) (2007) 597.

[59] Y.-K. Zhang, D. Zhang, J. Lin, Nonergodic solute transport in threedimensional heterogeneous isotropic aquifers, Water Resour. Res. 32 (9) (1996) 2955-2963.

[60] V. Kapoor, P. K. Kitanidis, Concentration fluctuations and dilution in aquifers, Water Resour. Res. 34 (5) (1998) 1181-1193.

[61] F. P. J. de Barros, A. Fiori, First-order based cumulative distribution function for solute concentration in heterogeneous aquifers: Theoretical analysis and implications for human health risk assessment, Water Resour. Res. 50 (5) (2014) 4018-4037.

[62] M. Moslehi, R. Rajagopal, F. P. J. de Barros, Optimal allocation of computational resources in hydrogeological models under uncertainty, Adv. Water Resour. 83 (2015) 299-309.

[63] C. V. Henri, D. Fernandez-Garcia, F. P. J. de Barros, Probabilistic human health risk assessment of chemical mixtures in heterogeneous aquifers: Risk statistics, hot spots and preferential flow channels, Water Resour. Res 51.

[64] C. V. Henri, D. Fernàndez-Garcia, F. P. J. de Barros, Assessing the joint impact of dnapl source-zone behavior and degradation products on the probabilistic characterization of human health risk, Adv. Water Resour. 88 (2015) 124-138. 
[65] D. Fernàndez-Garcia, G. Llerar-Meza, J. J. Gómez-Hernández, Upscaling transport with mass transfer models: Mean behavior and propagation of uncertainty, Water Resour. Res. 45 (10).

[66] L. Li, H. Zhou, J. J. Gómez-Hernández, Transport upscaling using multirate mass transfer in three-dimensional highly heterogeneous porous media, Adv. Water Resour. 34 (4) (2011) 478-489.

[67] S. P. Neuman, Blueprint for perturbative solution of flow and transport in strongly heterogeneous composite media using fractal and variational multiscale decomposition, Water Resour. Res. 42 (6).

[68] A. Bellin, A. E. Lawrence, Y. Rubin, Efficient monte carlo simulations of transport in heterogeneous formations by using block-scale dispersivity, in: Calibration and Reliability in Groundwater Modelling: A Few Steps Closer to Reality (Proceedings of ModelCARE 2002. Prague. Czech Republic, 17-20 June 2002), Vol. 277, IAHS PUBLICATION, 2003, pp. 89-94. 